\title{
Botánica Forense en Chile: El caso de Aristotelia chilensis (Molina) Stuntz y su potencial utilidad como especie bioindicadora forense
}

\section{Forensic Botany in Chile: The case of Aristotelia chilensis (Molina) Stuntz and its potential utility as a forensic bioindicator species}

\author{
Mario Romero-Mieres ${ }^{1 *}$, Gabriel Vivallo ${ }^{1}$, Gustavo Donoso ${ }^{1}$, Carlos Esse ${ }^{1}$, Ramiro Díaz ${ }^{1}$, Angélica \\ Francols $^{1}$, Jaime Solano ${ }^{1}$, Alexander Ortloff ${ }^{1}$, Sandra Albornoz ${ }^{1}$, Oriana Betancourt ${ }^{1}$, Ximena \\ Cofré ${ }^{1}$, Margarita Valdivia ${ }^{1}$, Juan Carlos de La Fuente ${ }^{2}$, Alejandra Figueroa ${ }^{2} \&$ Cristián Lizama ${ }^{2}$ \\ ${ }^{1}$ Facultad de Recursos Naturales, Universidad Católica de Temuco, Chile. \\ 2Laboratorio de Criminalística Regional Temuco, Policía de Investigaciones de Chile, Región de La Araucanía, Chile. \\ *maromero@uct.cl
}

\begin{abstract}
Aristotelia chilensis is mentioned for the first time for plants grown on pig carcasses decomposing. Its potential as a bioindicator species for the development of forensic botany in Chile and its importance in the reconstruction of criminal events are discussed.
\end{abstract}

La botánica forense es definida como el conocimiento de las características detalladas de las plantas, aplicado desde la perspectiva forense (Bock \& Norris 1997). Como rama de la botánica, es de reciente desarrollo e incorpora para su estudio otras disciplinas, como la palinología, dendrocronología, limnología, ecología y biología molecular (Dickison 2000, Coyle 2005, Craft et al. 2007, Fernández-Alonso et al. 2007). La identificación de plantas contribuye a determinar el origen geográfico de ellas y la relación entre la escena del crimen y el (los) sospechoso(s), así como también la posibilidad de relacionar la causa de muerte con la época del año en que ocurrió el crimen (Ferri et al. 2009, Coyle et al. 2005). Internacionalmente, ha sido de gran ayuda en diversos procedimientos forenses encaminados a rastrear y reconstruir eventos delictivos (Fernández-Alonso et al. 2007, Ferri et al. 2008, Aquila et al. 2014), siendo el secuestro y muerte del hijo de Charles Lindbergh en 1932 el primer caso en utilizar pruebas botánicas en procesos judiciales (Coyle et al. 2001).

En el año 2012, se inicia un proyecto pionero en Chile: "La botánica forense en la investigación policial" (FONDEF D11I1024). Uno de sus objetivos fue identificar la sucesión vegetal cadavérica asociada a cuerpos en distintos estados de descomposición bajo diferentes coberturas boscosas", es decir, qué especies de plantas se establecen sobre o alrededor de cuerpos abandonados en bosques o sitios eriazos y que con el tiempo modifican el lugar a partir del crecimiento y desarrollo de ellas. Estudios de este tipo son inéditos en Chile, ya que hasta ahora sólo existen antecedentes sobre otra disciplina: entomología forense (Ortloff et al. 2012, 2013).
El objetivo de este trabajo es aportar antecedentes sobre botánica forense en Chile, particularmente de la presencia de plántulas de Aristotelia chilensis (Molina) Stuntz en el proceso de descomposición de cadáveres de cerdo (Sus scrofa domesticus Linnaeus), en diferentes ecosistemas forestales del sur del país. Cuerpos de cerdos han sido utilizados para estudios de descomposición por varios investigadores dada la similitud que este proceso tiene con la descomposición de cadáveres humanos (Van Belle et al. 2009, Schotsmans et al. 2011). Se discute en el contexto de la botánica forense la autoecología de la especie y su potencial rol como bioindicador vegetal en el rastreo y reconstrucción de eventos delictivos.

Aristotelia chilensis, conocido comúnmente como Maqui o Clon, es un árbol endémico de los bosques subantárticos de Chile y Argentina (Rodríguez et al. 1983). Pertenece a la familia Elaeocarpaceae Juss. y se distribuye en Chile desde la provincia de Limarí $\left(21^{\circ} \mathrm{S}\right)$ hasta la provincia de Aysén $\left(43^{\circ} \mathrm{S}\right)$, tanto por el valle central como en ambas cordilleras, y desde el nivel de mar hasta los 2.500 m s.n.m. (Rodríguez et al. 1983, Donoso \& Ramírez 1997). Es una especie dioica, de 4-5 m promedio de altura, de hojas simples, opuestas dispuestas en cruz, coriáceas, oval-lanceoladas, con borde aserrado, ápice agudo y de color verde claro brillante. Sus peciolos, al igual que las ramas nuevas, son de color rojizo (Rodríguez et al. 1983, Hoffman 1997, Donoso \& Ramírez 1997, Riedemann \& Aldunate 2003, Rodríguez et al. 2005). Posee bayas pequeñas de color morado oscuro a negro brillante, las que contienen entre dos a cuatro semillas (Montenegro 2000). Estas bayas son comestibles y han sido 
utilizadas desde hace muchos años para la preparación de jugos, mermeladas, tinturas y bebidas alcohólicas (Donoso \& Ramírez 1997, Montenegro 2000).

Se seleccionaron cuatro sitios de estudio, a partir de la representatividad que estos tienen en la Región de La Araucanía: (1) bosque siempreverde (519 m s.n.m.), (2) bosque caducifolio (580 m s.n.m.), (3) plantación de Eucalyptus nitens Maiden (476 m s.n.m.) y (4) plantación de Pinus radiata D. Don (291 m s.n.m.) (Tabla I); los cuales se encuentran ubicados en el cordón montañoso HuimpilÑielol, en los predios La Envidia y El Arrejón, de propiedad de Forestal Mininco S.A. (Fig. 1).

En cada sitio de estudio se establecieron parcelas cercadas de $400 \mathrm{~m}^{2}$. En cada una de ellas fueron ubicadas seis subparcelas de $1 \mathrm{~m}^{2}$, en las cuales se llevaron a cabo los ensayos con cadáveres de cerdos (uno por subparcela), de 20 $\mathrm{kg}$ cada uno. En cada parcela se realizó un censo florístico por estratos y se determinaron coberturas estimadas para cada estrato considerando el método de Braun-Blanquet (1979), pre y post establecimientos de los ensayos (Tabla I). Asimismo, en cada subparcela se registró la composición florística pre y post postura de los cadáveres de cerdo. Se realizaron censos florísticos cada 30 días en cada subparcela y por cada sitio de estudio, desde marzo de 2013 a marzo de 2015. En este periodo además se midieron variables ambientales por sitio y por cada subparcela, incluyendo las temperaturas de suelo y de cadáver, más el registro de la tasa de descomposición.

En el periodo indicado se observó en cada subparcela una variación en la flora aledaña a los cadáveres de cerdos. Las especies que inicialmente se encontraban en el lugar, como plántulas de Gevuina avellana Molina, Lomatia dentata (Ruiz et Pav.) R. Br., Pinus radiata D. Don, Prunella vulgaris L., Agrostis capillaris L., Boquila trifoliolata (DC.) Decne. y Nothofagus obliqua (Mirb.) Oerst., no continuaron su crecimiento y al cabo de tres semanas comenzaron a morir, mismo tiempo en que se inicia el proceso de generación de fluidos desde el cadáver. En ninguna de las subparcelas, en todos los sitios, se registró plántulas y/o brotes vegetativos de $A$. chilensis previa instalación de los ensayos.

Iniciada la retirada invernal y coincidente con el aumento de las temperaturas en el área (agosto de 2013), la única especie que se estableció naturalmente por medio de plántulas, tanto alrededor como entre los restos de los cuerpos de cerdos en descomposición, fue $A$. chilensis (en cada subparcela de los cuatro sitios) (Fig. 2). Algunas

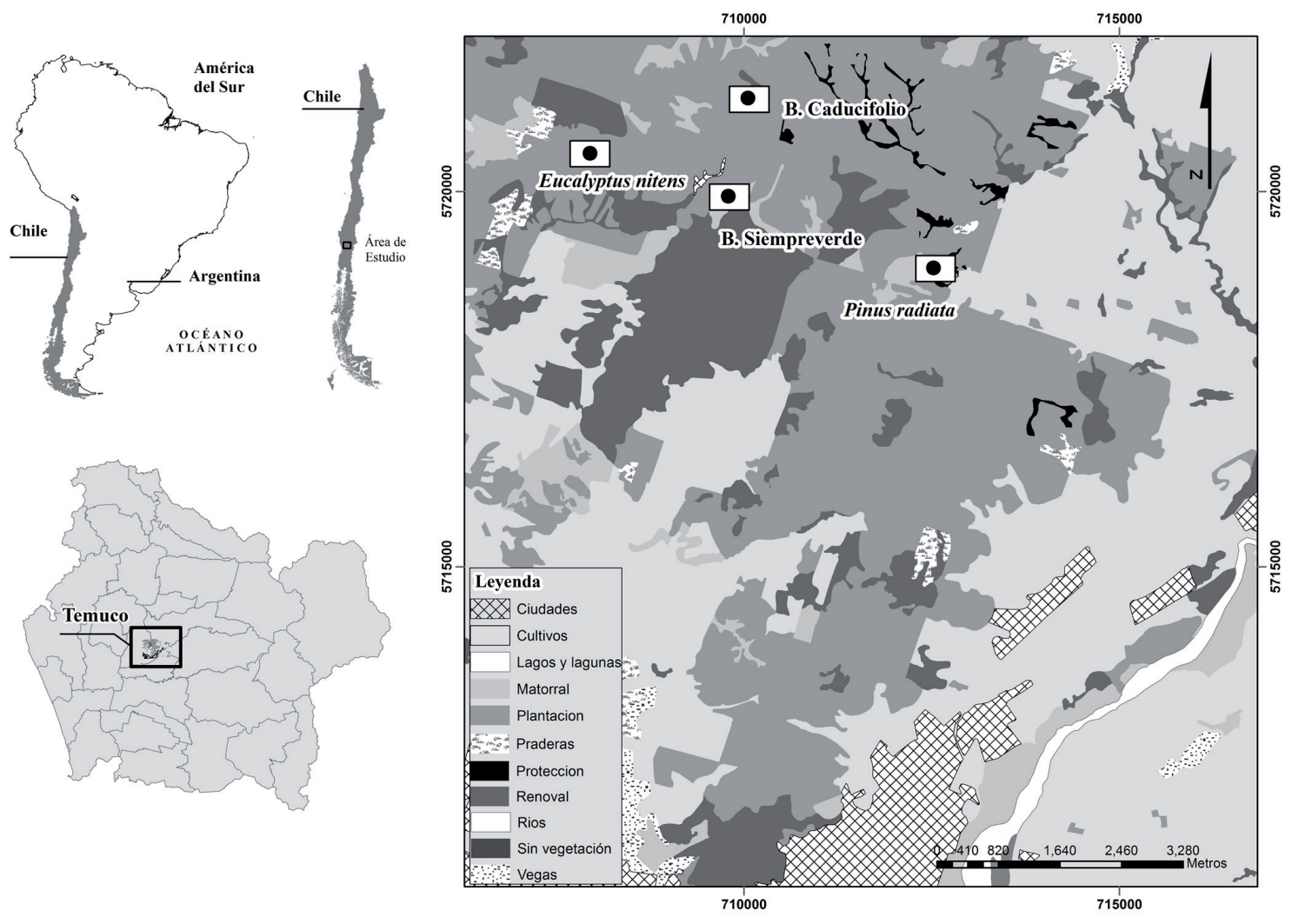

Figura 1. Área de estudio. Cordón montañoso Huimpil-Ñielol y ubicación de sitios.

FIGURE 1. Study area. Huimpil-Ñielol range and study sites. 
especies que siguieron a $A$. chilensis en el establecimiento del primer año (fines de noviembre 2013), como Nothofagus obliqua, Lomatia dentata, Rhamnus diffusus Clos y algunas representantes de la familia Poaceae, no prosperaron.
Aristotelia chilensis ha prolongado su crecimiento y desarrollo hasta la actualidad. Sólo durante la segunda temporada, otras especies han comenzado a poblar cada subparcela, tanto por semillas como vegetativamente.

Tabla I. Características botánicas de los sitios de estudio. Nomenclatura según Zuloaga et al. (2008), excepto Nothofagus obliqua (Rodríguez \& Quezada 2003).

TABLE I. Botanical characteristics of the study area. Nomenclature according Zuloaga et al. (2008), except Nothofagus obliqua (Rodríguez \& Quezada 2003).

\begin{tabular}{|c|c|c|}
\hline Sitio/ $\mathrm{N}^{\mathrm{o}}$ ESPeCies & Principales esPeCies & $\begin{array}{c}\text { CoBERTURA ARBÓREA } \\
(\%)\end{array}$ \\
\hline $\begin{array}{l}\text { Bosque siempreverde } \\
/ 27 \mathrm{spp} .\end{array}$ & $\begin{array}{l}\text { Aextoxicon punctatum Ruiz et Pav., Aristotelia chilensis, Blechnum cordatum (Desv.) } \\
\text { Hieron., Campsidium valdivianum (Phil.) Skottsb., Chusquea macrostachya Phil., } \\
\text { Dasyphyllum diacanthoides (Less.) Cabrera, Eucryphia cordifolia Cav., Gevuina } \\
\text { avellana, Myrceugenia planipes (Hook. et Arn.) O. Berg }\end{array}$ & 80 \\
\hline $\begin{array}{l}\text { Bosque caducifolio } \\
/ 23 \mathrm{spp} \text {. }\end{array}$ & $\begin{array}{l}\text { Blechnum hastatum Kaulf., Aristotelia chilensis, Cissus striata Ruiz et Pav., Lapageria } \\
\text { rosea Ruiz et Pav., Nothofagus obliqua, Persea lingue Nees, Podocarpus salignus D. } \\
\text { Don, Rhamnus diffusus, Rhaphithamnus spinosus (Juss.) Moldenke }\end{array}$ & 75 \\
\hline $\begin{array}{l}\text { Plantación de } \\
\text { Eucalyptus nitens } \\
\text { /24 spp. }\end{array}$ & $\begin{array}{l}\text { Acaena ovalifolia Ruiz et Pav., Aristotelia chilensis, Boquila trifoliolata, E. nitens, } \\
\text { Rubus constrictus P.J. Müll. et Lefèvre }\end{array}$ & 60 \\
\hline $\begin{array}{l}\text { Plantación de Pinus } \\
\text { radiata } \\
/ 12 \text { spp. }\end{array}$ & $\begin{array}{l}\text { Agrostis capillaris, Aristotelia chilensis, Arrhenatherum elatius (L.) P. Beauv. ex J. } \\
\text { Presl \& C. Presl, Boquila trifoliolata, Cissus striata, Rubus constrictus, Pinus radiata }\end{array}$ & 60 \\
\hline
\end{tabular}

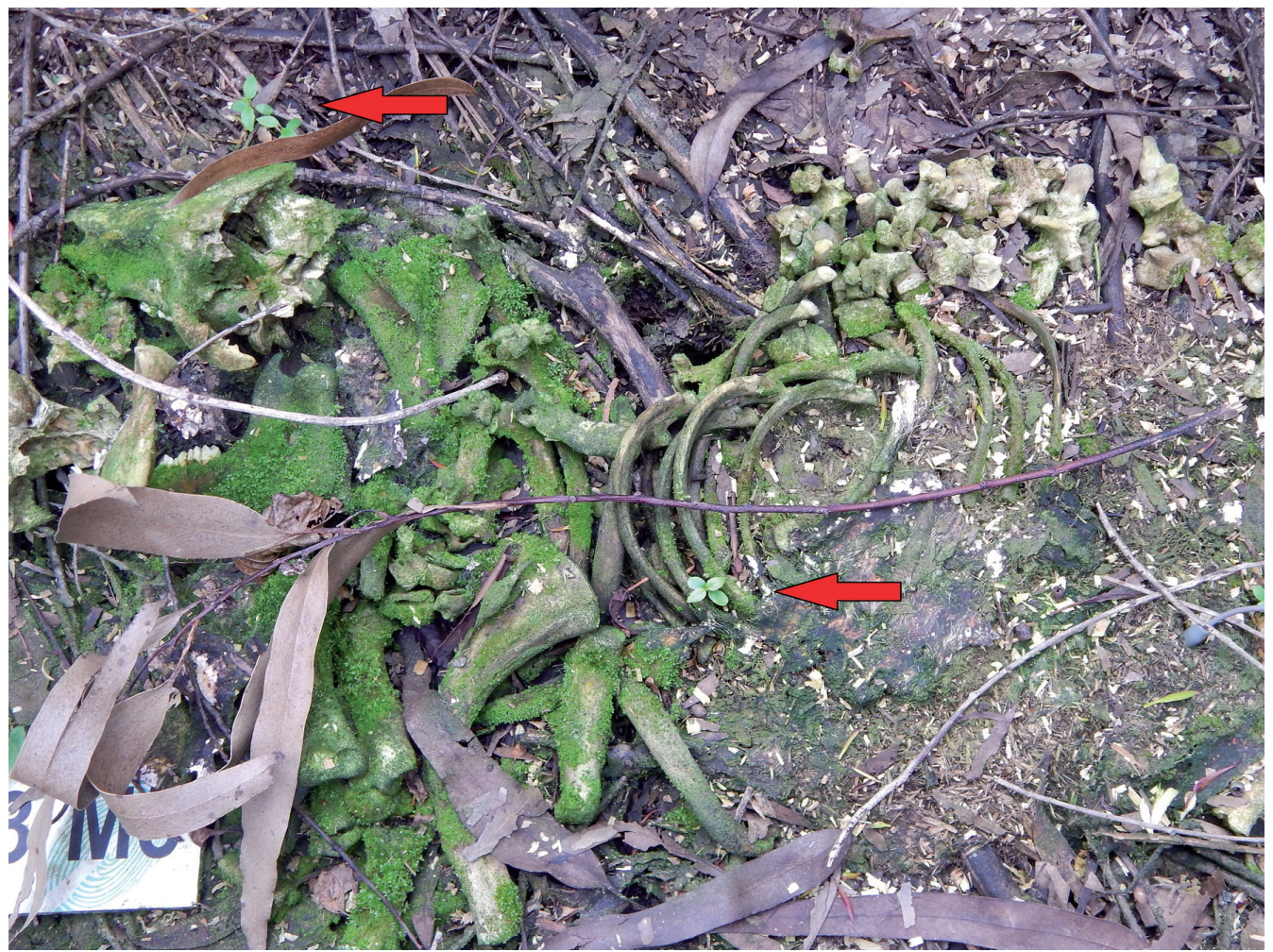

Figura 2. Plantas de $A$. chilensis sobre cadáveres de $S$. scrofa (flechas rojas).

Figure 2. Aristotelia chilensis plants on Sus scrofa carcasses (red arrows). 
Desde el punto de vista de la dinámica vegetacional en áreas boscosas, Picket (1980) y Oliver \& Larson (1990) señalan que los procesos sucesionales son iniciados por diferentes organismos, entre los que se encuentran las plantas vasculares pioneras. Y, sucesionalmente, el proceso de reemplazo de especies y el cambio en el desempeño de los individuos a través del tiempo en un mismo espacio, genera una nueva composición florística, por lo que a escala de paisaje puede ser apreciable y descriptiva, y en casos forenses potencialmente relevantes (por ejemplo: fosas clandestinas, desplazamiento de cadáveres). Aristotelia chilensis es una especie pionera, intolerante a la sombra, que se caracteriza por ocupar áreas que han sido alteradas antrópicamente (San Martín et al. 1991, Ramírez \& San Martín 2005, Muñoz \& González 2006). Es una especie típica de etapas sucesionales tempranas y forma parte de comunidades dominadas por bosques secundarios de Nothofagus y bosque siempreverde (Donoso 1994), tal y como se aprecia en los sitios de bosque estudiados (Tabla I). Además, en el bosque, las condiciones de humedad, disponibilidad de nutrientes y principalmente de luz, favorecen el establecimiento de plántulas, siendo esto relevante en la mantención o cambio de la densidad de las especies que dominan una determinada comunidad vegetal (White 1979, Veblen 1992).

Para la botánica forense, observar y registrar variaciones post mortem a nivel de micropaisaje en condiciones boscosas resulta del todo significativo, donde la presencia de $A$. chilensis en las condiciones ya detalladas contribuye a ello. El crecimiento y desarrollo de esta especie en condiciones de descomposición cadavérica resulta en una novedad botánica, realzando su condición de especie pionera y de gran capacidad adaptativa en lugares que han sido alterados por el ser humano. En cada sitio de estudio los ejemplares de $A$. chilensis no presentaban desarrollo de frutos, por lo que la procedencia de los propágulos en los cadáveres de cerdo se presume de poblaciones distantes del área estudiada. Esto, en una investigación policial, resulta relevante en la reconstrucción de eventos delictivos.

Estos antecedentes sugieren el potencial rol bioindicador de $A$. chilensis en el marco de la botánica forense, ya que al ser la primera especie en emergery mantenerse en las condiciones expuestas y bajo diferentes ecosistemas forestales, genera un cambio perceptible a escala de micropaisaje a los pocos meses de iniciado un proceso de descomposición cadavérica. Esto permitirá a los peritos forenses sumar otra variable más a las causas que llevan a cabo en condiciones similares en el sur de Chile, contribuyendo a la vinculación entre la evidencia forense y el sitio del suceso.

\section{AGRADECIMIENTOS}

Los autores agradecen al proyecto "La Botánica Forense en la Investigación Policial” (FONDEF D11I1024) por el financiamiento de esta contribución, a Forestal Mininco S.A. por la facilidad de acceso y trabajo en sus predios, y a la Ingeniero Agrónomo Valeria Sanhueza por su apoyo en terreno.

\section{BIBLIOGRAFÍA}

Aquila, I., F. Ausania, C. Di Nunzio, A. Serra, S. Boca, A. Capelli, P. Magni \& P. Ricci. 2014. The role of forensic botany in crime scene investigation: case report and review of literatura. Journal of Forensic Sciences 59(3): 820-4.

Bock, J. \& D. NorRIs. 1997. Forensic botany: an under-utilized resource. Journal of Forensic Sciences 42(3): 364-367.

Braun-Blanquet, J. 1979. Fitosociología. Base para el estudio de las comunidades vegetales. Editorial Blume, Madrid. 820 pp.

Craft, K., J. Owens \& M. Ashley. 2007. Application of plant DNA markers in forensic botany: Genetic comparison of Quercus evidence leaves to crime scene trees using microsatellites. Forensic Science International 165: 64-70.

Coyle, H.M. 2005. Forensic Botany. Principles and applications to criminal casework. CRC Press, Florida. 318 pp.

Coyle, H.M., C. Ladd, T.M. Palmbach \& H.C. Lee. 2001. The green revolution: botanical contributions to forensics and drug enforcement. Croatian Medical Journal 42: 340-5.

Coyle, H.M., C.L. Lee, W.Y. Lin, H.C. Lee \& T.M. Palmbach. 2005. Forensic botany: using plant evidence to aid in forensic death investigation. Croatian Medical Journal 46(4): 606-12.

Dickison, W. 2000. Integrative plant anatomy. Academic Press, San Diego. 533 pp.

Donoso, C. 1994. Bosques templados de Chile y Argentina. Editorial Universitaria, Santiago de Chile. 484 pp.

Donoso, C. \& C. Ramírez. 1997. Arbustos nativos de Chile. Guía de reconocimiento. Marisa Cúneo Ediciones, Valdivia. 119 pp.

Fernández-Alonso, J.L., A. Galindo \& J.M. Idrobo. 2007. Las plantas como evidencia legal. Desarrollo de la botánica forense en Colombia. Revista de la Academia Colombiana de Ciencias Exactas, Físicas y Naturales 31: 181-198.

Ferri, G., M. Alù, B. Corradini, A. Angot \& G. Beduschi. 2008. Land plants identification in forensic botany: Multigene barcoding approach. Forensic Science International, Genetics Supplement Series 1: 593-595.

Ferri, G., M. Alù, B. Corradini \& G. Beduschi. 2009. Forensic botany: species identification of botanical trace evidence using a multigene barcoding approach. International Journal of Legal Medicine 123: 395-401.

Hoffmann, A. 1997. Flora silvestre de Chile, zona araucana. Cuarta edición revisada. Ediciones Fundación Claudio Gay, Santiago. 258 pp.

Montenegro, G. 2000. Chile, nuestra flora útil. Guía de uso apícola, medicinal, folklórica, artesanal y ornamental. Editorial Universidad Católica de Chile, Santiago. 267 pp.

Muñoz, A. \& M. GonzÁlez. 2006. Aristotelia chilensis (Mol) Stuntz. En: C. Donoso (ed.), Las especies arbóreas de los bosques templados de Chile y Argentina. Autoecología, pp. 166-172. Marisa Cuneo Ediciones, Valdivia.

Oliver, C. \& B. Larson. 1990. Forest Stand Dynamics. McGraw- 
Hill New York. 520 pp.

Ortloff, A., P. Peña \& M. Riquelme. 2012. Preliminary study of the succession pattern of necrobiont insects, colonising species and larvae on pig carcasses in Temuco (Chile) for forensic applications. Forensic Science International 222: e36-e41.

Ortloff, A., A. Jara, S. Albornoz, R. Silva, M. Riquelme \& P. PeÑa. 2013. Primer reporte en Chile de Chrysomya albiceps (Diptera: Calliphoridae) en evidencia entomológica forense. Archivos de Medicina Veterinaria 45(1): 83-89.

PiCKet, S. 1980. Non-equilibrium coexistence of plants. Bulletin of Torrey Botanical Club 107: 238-248.

Ramírez, C. \& C. San Martín. 2005. Asociaciones vegetales de la Cordillera de la Costa de la Región de Los Lagos. En: Smith-Ramírez et al. (eds.), Historia, biodiversidad y ecología de los bosques costeros de Chile, pp. 206-223. Editorial Universitaria, Santiago, Chile.

Riedemann, P. \& G. Aldunate. 2003. Flora nativa de valor ornamental. Identificación y propagación. Chile - Zona Sur. Primera edición. Editorial Andrés Bello, Santiago de Chile. 516 pp.

Rodríguez, R., O. Matthei \& M. Quezada. 1983. Flora Arbórea de Chile. Editorial Universidad de Concepción, Concepción. 408 pp.

Rodríguez, R. \& M. Quezada. 2003. Fagaceae: Nothofagus
Blume, nom. cons. En: C. Marticorena \& R. Rodríguez (eds.), Flora de Chile. Vol 2(2). Berberidaceae-Betulaceae. Ediciones Universidad de Concepción, Concepción. 93 pp. Rodríguez, R., E. Ruiz \& J.P. Elissetche. 2005. Árboles en Chile. Editorial Universidad de Concepción, Concepción. 183 pp.

San Martín, C., C. Ramírez, H. Figueroa \& N. Ojeda. 1991. Estudio sinecológico del bosque de roble-laurel-lingue del centro-sur de Chile. Bosque 12(2): 11-27.

Schotsmans, E.M., W.V. Voorde, J.D. Winne \& A.S. Wilson. 2011. The impact of shallow burial on differential decomposition to the body: a temperate case study, Forensic Science International 206 (1-3): e43-e48.

Van Belle, L.E., D.O. CARTer \& S.L. Forbes. 2009. Measurement of ninhydrin reactive nitro-gen influx into gravesoil during aboveground and belowground carcass (Sus domesticus) decomposition. Forensic Science International 193: 7-41.

Veblen, T. 1992. Regeneration dynamic. In: D.C. Glen-Lewin et al. (eds.), Plant Succession: theory and prediction, pp. 152187. Champan \& Hall. Cambridge, United Kingdom.

White, P. 1979. Pattern, process and natural disturbances in vegetation. The Botanical Review 45: 229-299.

Zuloaga, F., O. Morrone \& M. Belgrano. 2008. Catálogo de las plantas vasculares del Cono Sur (Argentina, sur de Brasil, Chile, Paraguay y Uruguay). , Missouri Botanical Garden Press, Saint Louis. 3348 pp.

Recibido: 14.04 .15

Aceptado: 08.03.16 